\title{
3 Stage 2 Switch Application for Transcranial Magnetic Stimulation
}

\author{
Dong-Ho Ha ${ }^{1}$, Whi-Young Kim² ${ }^{2}$ and Sun-Seob $\mathrm{Choi}^{3 *}$ \\ ${ }^{1}$ Department of Radiology/Neuro Radiology Section, Dong-A University Medical Center, Busan 602-715, Korea \\ ${ }^{2}$ Department of Biomedical Engineering, Dong-ju College University, Busan 602-715, Korea \\ ${ }^{3}$ Department of Radiology/Neuro Radiology Section, Dong-A University Medical Center, Busan 602-715, Korea
}

(Received 12 April 2011, Received in final form 15 September 2011, Accepted 19 September 2011)

\begin{abstract}
Transcranial magnetic stimulation utilizes the method of controlling applied time and changing pulse by output pulse through power density control for diagnosis purposes. Transcranial magnetic stimulation can also be used in cases where diagnosis and treatment are difficult since output pulse shape can be changed. As intensity, pulse range, and pulse shape of the stimulation pulse must be changed according to lesion, the existing sine wave-shaped stimulation treatment pulse poses limitations in achieving various treatments and diagnosis. This study actualized a new method of transcranial magnetic stimulation that applies a 3 Stage 2 Switch( power semiconductor 2EA) for controlling pulse repetition rate by achieving numerous switching control of stimulation coil. Intensity, pulse range, and pulse shape of output can be freely changed to transform various treatment pulses in order to overcome limitations in stimulation treatment presented by the previous sine wave pulse shape. The method of freely changing pulse range by using 3 Stage 2 Switch discharge method is proposed. Pulse shape, composed of various pulse ranges, was created by grafting PFN (Pulsed Forming Network) through AVR AT80S8535 one-chip microprocessor technology, and application in transcranial magnetic stimulation was achieved to study the output characteristics of stimulation treatment pulse according to delaying time of the trigger signal applied in section switch.
\end{abstract}

Keywords : stimulation, pulse, 3 Stage-2 Switch multi, discharge, treatment

\section{Introduction}

Research is actively being conducted on magnetic field generation using stimulation coils and generation device of parts that can continuously generate a strong, time varying magnetic field [1-3]. Formation of electric field by eddy current is the basic principle of magnetic stimulation, where an extremely strong current pulse flows into a stimulation generation coil for generating a magnetic field that sufficiently stimulates the human neuromuscular system by way of a time varying magnetic field [5]. The intensity of the electric field that generates the time varying magnetic field is proportional to the time change rate of the magnetic field, and strong pulse stimulation can be triggered according to duration of time varying magnetic field $[4,5]$. To operate, the magnetic field is emitted from the outside according to the law of induction. Thus, as it does not directly possess basic power

*Corresponding author: Tel: +82-51-240-2982

Fax: +82-51-253-4931, e-mail: sschoi317@yahoo.co.kr consumption, the lifespan is longer than the traditional load and as a result there is a large advantage presented in terms of maintenance $[4,6,7]$. Research on commercialization of the stimulation coil is currently being conducted in various advanced nations, and its use is being gradually expanded for treatment and diagnosis [8,9]. As voltage, current peak point of main circuit largely changes from coil operation to magnetic field charge in stimulation coil, driving circuit that satisfies stimulation coil operation and magnetic field charge is $[4,11]$. The operating frequency of the stimulation coil a consideration in terms of energy efficiency flowing from the coil, and coupling efficiency changes according to operating frequency specifically in open ferrite core stimulation coils [6,7]. It can be known that high-frequency voltage of operating frequency is inevitable in stimulation coil operation [12]. The method for creating a flowing large sine-wave current using a discharge circuit, which forms inductance of the capacitor and coil as a resonance circuit, is used in when discharging a high-voltage charge in large-sized capacitors as a stimulation coil to generate a magnetic field. The system 
to allowing the flow of a consistent discharge current of sine-wave form in various patients is the trend. This study used the 3 Stage-2 Switch discharge method to develop pulse formation technology for precisely controlling and creating various shapes of current pulse stimulation coil that can create stimulation efficiency according to patient symptoms [4]. The main circuit was used as a Six-mesh network method composed of LC circuit network to gain sine waves that possess $1 \mathrm{~ms}$ pulse range and similar output throughout the entire pulse cycle, while the superposition circuit was composed of a Three-mesh network to create sine-wave of $0.2 \mathrm{~ms}$ pulse range. The delay time of the sine-wave shaped superposition pulse gained from the superposition circuit is freely changed by externally attached resistance $\mathrm{R}$ and condenser $\mathrm{C}$ of one-shot multivibrator, thus achieving diverse control of the current pulse shape from the stimulation coil for appropriately recommending and enhancing quality of treatment [11]. Output of the stimulation coil during application of equivalent input energy without superposition and superposed stair-type pulse were compared and studied, and stimulation coil outputs according to stair-type pulse shape were compared and examined to summarize results regarding the conditions for gaining improved efficiency. As the previous transcranial magnetic stimulation is composed of a complex control circuit and uses an expensive high- frequency condenser during high-repetition operation, a sufficient power device is required. Here, we propose a new method and power device for efficiently executing a more compact, economic output control of stimulation pulse than previous power devices. Output control characteristics of transcranial magnetic stimulation according to change in pulse repetition rate were researched through the application of an AVR one chip microprocessor, which can precisely control current pulse output, and the 3 Stage 2 Switch charge/discharge method, which uses an inexpensive $60 \mathrm{~Hz}$ condenser.

\section{Circuit Topology}

Treatment effect is outstanding as magnetic field is harmless to human body and can impact neural tissue and muscular tissue through deep penetration. Also, stresstriggered depression, insomnia, and bipolar disorder can be cured through magnetic field treatment of neural tissue and muscular tissue.

To achieve deep penetration in human tissue, a waveform with narrow pulse width and fast rise time is highly preferred. It is most suitably used as a device for executing magnetic treatment of the human body with less noise and heat occurrence. The biphasic type of waveform is regarded as the most appropriate in this scenrio, and all

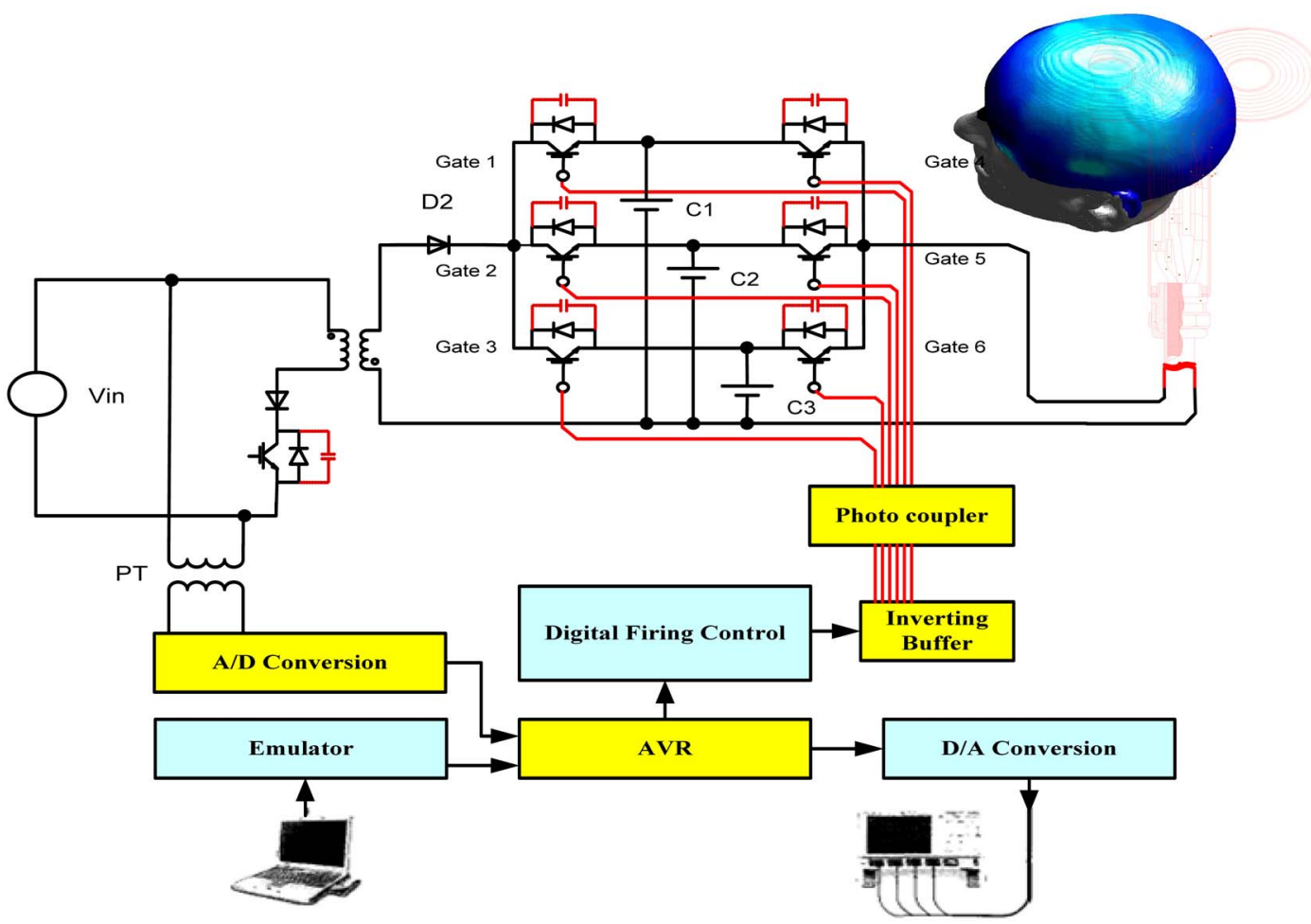

Fig. 1. (Color online) A new method of transcranial magnetic stimulation that applies 3 Stage-2 Switch to control pulse repetition rate of stimulation coil through numerous switching controls. 
domestic and foreign PMF (Pulsed Magnetic Field) types use the Biphasic type. The existing method has several weaknesses including rapidly decreased energy transmission efficiency in response to increase in frequency (Pulse repetition rate), and is also disadvantageous in that various changes of pulse shape cannot be achieved. As IGBT (Insulate Gated Bipolar Transistor) can be turned on and off, pulse increase time can be shortened and created into various shapes, while energy transmission loss can be decreased as there is an increase in pulse repetition rate. Fig. 1 presents a new method of transcranial magnetic stimulation that applies 3 Stage 2 Switch for controlling pulse repetition rate through various switching controls. The entire system is largely composed of a stimulation coil, pulse power device, control circuit, and control section using AVR one chip microprocessor. In order to stimulate nerves through magnetic pulse, electric field induced by time-varying electric field must be composed of a size that can stimulate nerves. An electric field composed of dozens of $\mathrm{V} / \mathrm{m}$ must be induced in the neural area, and switching of 1-2 Tesla electric field must be achieved within a short period of time $(200 \mu \mathrm{Sec})$ from stimulation coil shape and epidermis to neural area. Thousands of amperes currents must be flown in stimulation coil that is comprised of a $0.5 \mathrm{~cm}$ diameter and dozens of coils in order to achieve formation of the appropriate magnetic field. As a method for instantaneously applying a large quantity of current in the stimulation coil, a capacitor is polarized to temporarily discharge in the stimulation coil. When current flows in the coil wire, a magnetic field is generated near the a coil wire according to Fleming's Right Hand Rule, and an electric field is induced in the direction vertical to the magnetic field. Eddy current is generated within the conductor when a time varying magnetic field is applied in the surrounding area of electric conductors, such as in the case of metals. Average electric conductivity of the human body is approximately 0.42 Simens $/ \mathrm{m}$. Although electric conductivity is very low when compared with high-quality conductors, such as metals, it possesses the properties of a conductor. Thus, eddy current can be induced within the human body along with regular electric conductor through application of a timevarying magnetic field in surrounding area of human body. Eddy current induced by a time-varying magnetic field uses electrodes within the human body to produce the effect of directly injecting current. Fig. 2 operates 3 Stage 2 Switch for controlling pulse repetition rate through multiple switching controls and presents AVR one chip microprocessor (AT90S8535) of ATMEL for controlling various signals. In Fig. 1 we used a copper pipe with an internal diameter of $6.6 \mathrm{~mm}$ and an external diameter 8.6 $\mathrm{mm}$ in stimulation coil. It is primarily used to withstand

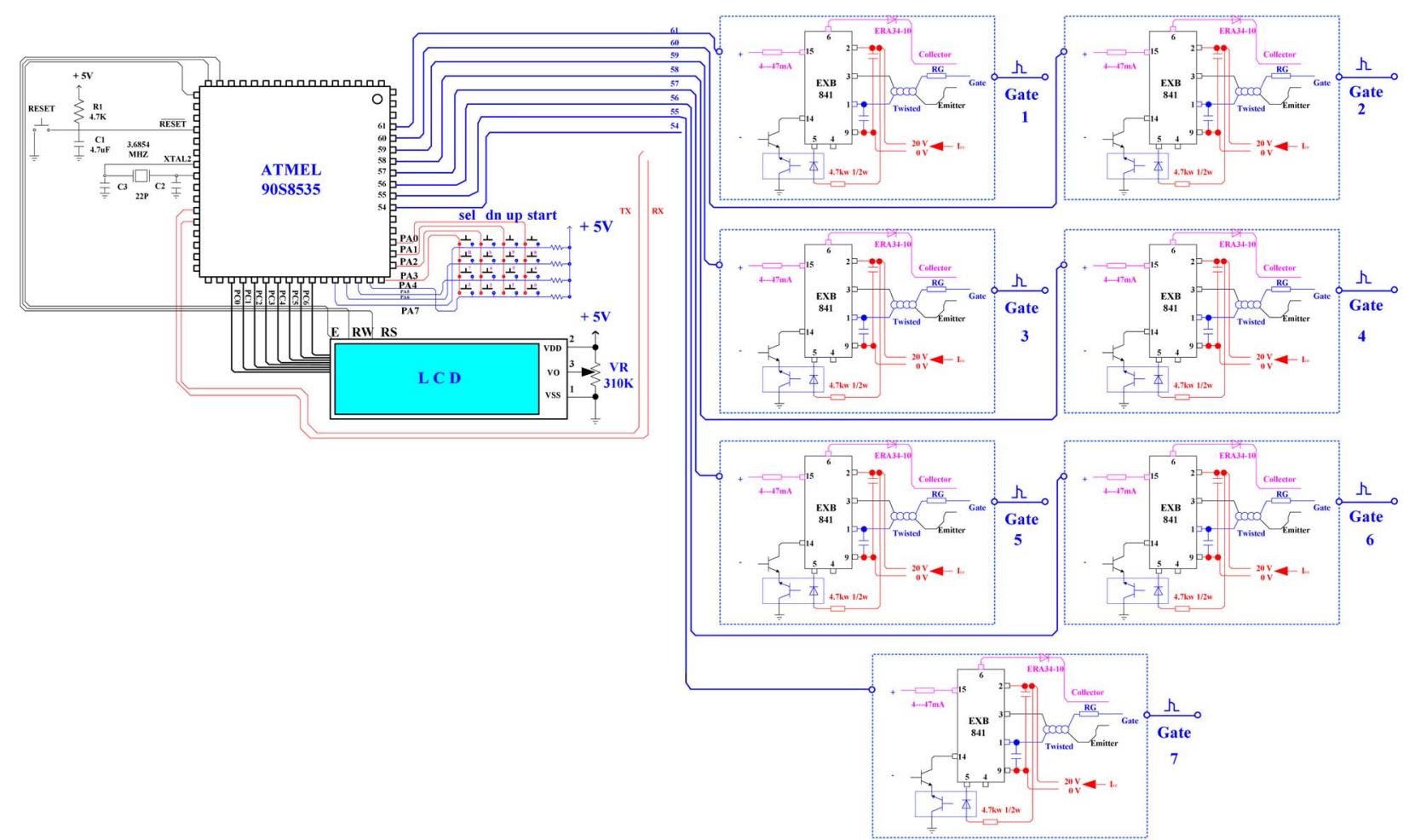

Fig. 2. (Color online) AVR-one-chip-microprocessor (AT90S8535/ATMEL) for operating 3 Stage-2 Switch to control pulse repetition rate through switching gear and control various signals. 
voltage of $1,440 \mathrm{~V}$, thickness ( $\mathrm{t}$ ) of $0.22 \mathrm{~mm}$ through glass fiber and copper pipe film to prevent barriers, such as wire interference, and secondarily used material of withstand voltage of $4,400 \mathrm{~V}$, thickness (t) to achieve complete shielding. Total length of the stimulation coil used in this study was $3.3 \mathrm{~m}$. The initial section was pressed as $11.3 \mathrm{~cm}$, the end section was pressed as $55.3 \mathrm{~cm}$, while the end section was secondarily pressed as $5.3 \mathrm{~cm}$. Material durability is strengthened by executing heat treatment (hardening) in $670^{\circ} \mathrm{C}$ for 13 minutes in total area, 8 minutes in end section for various uses Panels of $4.44 \mathrm{~cm}$ and $5.5 \mathrm{~cm}$ were added during press. Figure-of-eight coil probe used Litz wire (number of lead wires: 150 strands) to connect two helix types and produce 8-shaped form. Total number of turn is $9.9 \mathrm{~mm}$, and diameter is $77 \mathrm{~mm}$. Inductance value of coil is approximately $8.8 \mu \mathrm{H}$, and maximum magnetic field intensity is 1.35 Tesla. Stimulation coils can be used when executing concentrated treatment in the head or particular areas. In particular, adjustment of intensity, frequency, train time, pause time, and treatment time must be considered when designing transcranial magnetic stimulation. It is important to use the 3 Stage 2 Switch discharge method to sequentially operate stimulation coil in power circuit.

\section{Power Supply and Control Circuit}

In Fig. 3 we show the monitoring screen by $\mathrm{VC}+$ (Microsoft, Visual C+), the operation of various devices of 3 Stage-2 Switch discharge method, and present signals controlling various signals. If designated pulse repetition rate of the main circuit is $100 \mathrm{~Hz}, 3$ condensers of $\mathrm{C} 1, \mathrm{C}$ 2, C 3 actually divide the repetition rate into 3 parts for operation. Thus, greater amount of energy is saved compared with operation of a single condenser with a pulse repetition rate of $100 \mathrm{~Hz}$. This is advantageous in that greater charging time of condenser can be acquired, therefore high-output can be gained without using the existing high-frequency condenser by charging/discharging commercial frequency capacity. The operating principle of the circuit is as follows. Initially, energy charged in gate 2 and gate 4 is turned-on; C 1 begins stimulation coil discharge and $\mathrm{C} 2$ charges as well, and energy charged in gate 1 and gate 6 is then turned-on, after which $\mathrm{C} 3$ begins stimulation coil discharge and $\mathrm{C} 1$ charge. Next, energy charged in gate 3 and gate 5 is turned-on and $\mathrm{C} 2$ begins stimulation coil discharge and C 3 charge. IGBT gate signal is controlled through the control circuit to repeat sequential charge/discharge according to the order above. Sequential charge/discharge method was selected for charge/discharge method of condenser for 3 Stage 2

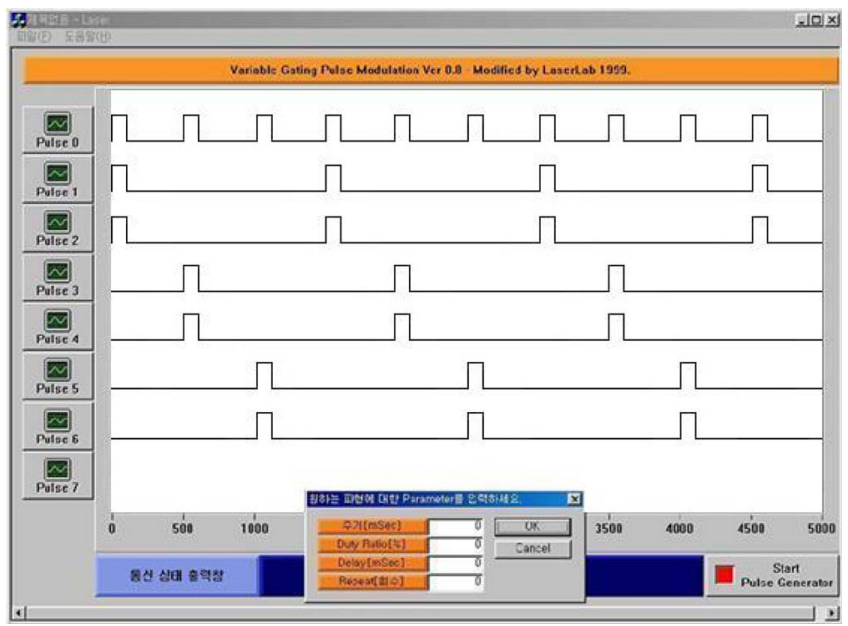

Fig. 3. (Color online) Monitoring screen processing by using the exchange information method, such as real-time data or control commands, as the block road of control circuit using microprocessor.

Switch discharge method. Control section was designed to sequentially execute charge/discharge in First-Charge, Last-Discharge form. In Fig. 3, one chip microprocessor and interface play an important role in computing through method of exchanging information, such as real-time data or control commands. Thus, control pulse is created on screen after perceiving signal entered in the computer through the RS-232 communication port from micro controller to check disorder and transmit to gate operation after passing the delay circuit through pulse generator. Through this, operation control signals required per IGBT module are sequentially controlled to charge/discharge condenser of main circuit. Fig. 4 presents the pulse for turning on IGBT in experiment. The main function in the microprocessor is to discriminate parameter transmitted from computer to input start signal and load information

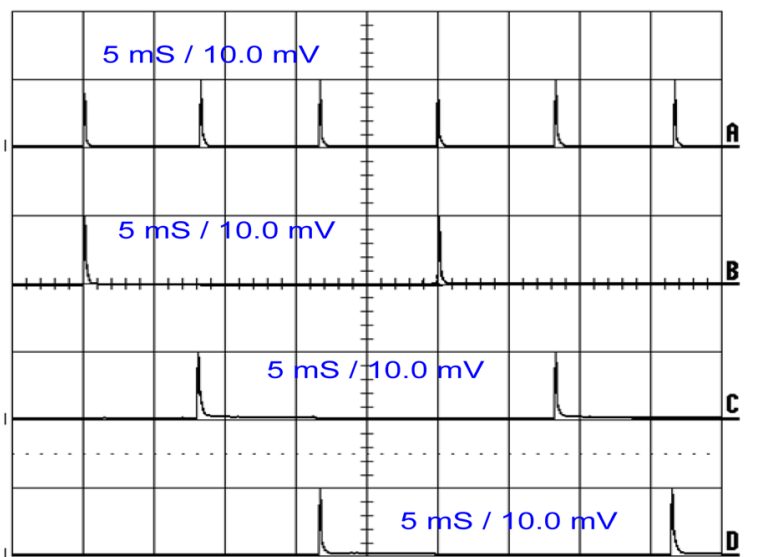

Fig. 4. (Color online) Pulse for turning on IGBT in experiment. 
of terminal in the register port for operation. It functions as a timer to generate pulse signal of transcranial magnetic stimulation. Signal is transmitted according to the number of controlled pulses. Signal stops and returns to communication initialization status after verification. Although the previous method processed limited saved file data, this method adopted the protocol of processing according to randomly varying value. Setup regarding cycle, duty rate, delay, and repetition rate can be achieved in screen composition composed of visual $\mathrm{C}++$ and operation regarding control screen input function, cognition function, and processing function modules, and pulse in each section can be achieved. It is composed of pulse 0 (gate 7), pulse 1 (gate 2), pulse 2 (gate 3), pulse 3 (gate 4 ), pulse 4 (gate 5 ), pulse 5 (gate 6 ) icons. Gate 1 , gate 2 , gate 3 , gate 4 , gate 5 , gate 6 , gate 7 are IGBTs in parallel sequential charge/discharge circuit, as shown in section 3 of Fig. 3. Pulse 0 (S7) operates as an IGBT trigger as the basic motion. Pulse 1 (gate 2), Pulse 2 (gate 4), Pulse 3 (gate 1), Pulse 4 (gate 6), Pulse 5 (gate 3), and Pulse 6 (gate 5) are operated such that settings of different values can be processed.

\section{Experiment and Result}

We pressed the start button on the screen to begin simulation of output waveform after completion of control data input. Input response signal of $90 \mathrm{~S} 8535$ was discriminated to check accurate transmission of pulse gene- ration data in AVR 90S8535 through communication verification processing function and display communication success/failure status on the screen. Communication protocol with 90S8535 was created to setup a communication environment and initialize the communication port through configuration. Data was transmitted to $90 \mathrm{~S} 8535$ through communication after completing setup of control waveform parameter pulse data released from each port from bit 0 to bit 7 of control pulse data. Command of introducing pulse generation of transcranial magnetic stimulation was transmitted to $90 \mathrm{~S} 8535$ in order to generate a control pulse based on output data currently saved in $90 \mathrm{~S} 8535$. Fig. 5, using the Maxwell 3D program is interpreting the results. Fig. 6 presents the current wave-

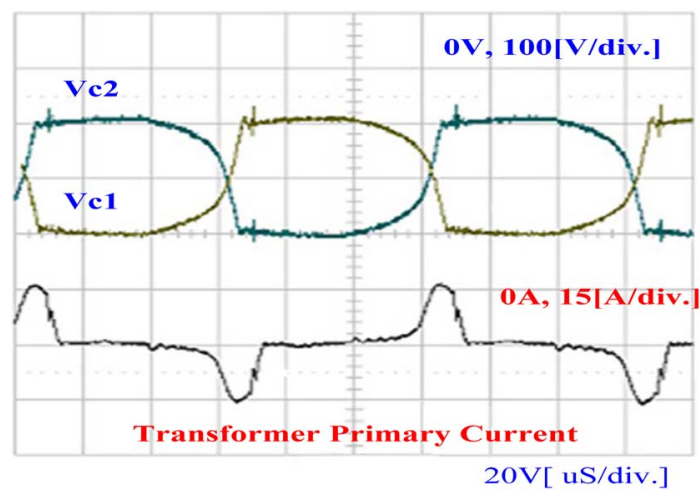

Fig. 6. (Color online) Current waveform flowing through the transformer primary and voltage of the resonant capacitor $\mathrm{V}_{\mathrm{C} 1}$ and $\mathrm{V}_{\mathrm{C} 2}$.

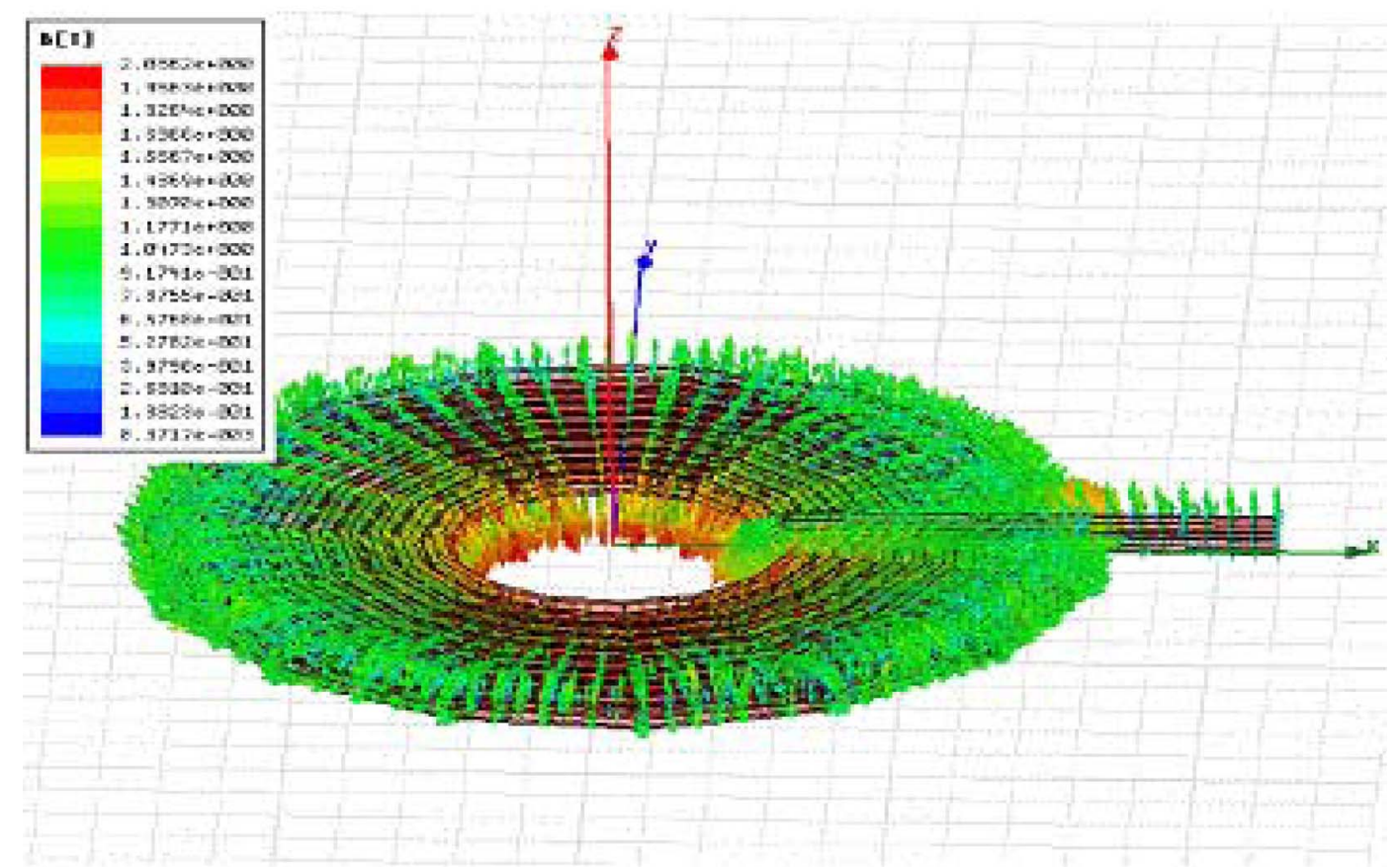

Fig. 5. (Color online) Using the Maxwell 3D program is interpreting the results. 


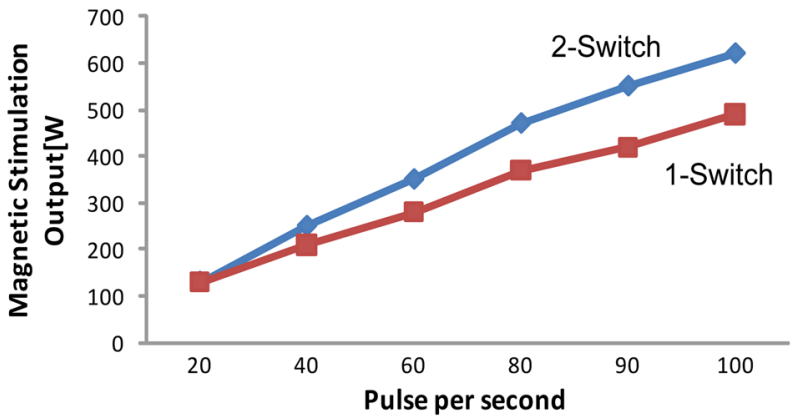

Fig. 7. (Color online) Relationship of transcranial magnetic stimulation output properties during application of $1000 \mathrm{~V}$ charged voltage using 3 Stage-2 Switch, and increase in repetition rate from $30 \mathrm{~Hz}$ to $100 \mathrm{~Hz}$ (20 Hz per increase).

form flowing through the transformer primary and voltage of the resonant capacitor $\mathrm{V}_{\mathrm{C} 1}$ and $\mathrm{V}_{\mathrm{C} 2}$. To simultaneously gain maximum pulse repetition rate of $100 \mathrm{~Hz}$ and minimize the effect of gate trigger pulse in turn-off time of IGBT, all sine-wave gate trigger pulses were filtered through a differentiating circuit to convert into waveforms with a short pulse range. As the converted waveform of short pulse range is too weak to be used as an IGBT gate operation current, high-speed switching transistor was used to amplify current and voltage. IGBT was sequentially turned on as signals are each drived at a regular cycle. The stimulation coil was not damaged and stable output characteristics we able to be displayed through execution of safe operation without under damping. Fig. 7 presents output characteristics of transcranial magnetic stimulation when repetition rate was increased from 30 $\mathrm{Hz}$ to $100 \mathrm{~Hz}$ (20 Hz per increase) during application of $1000 \mathrm{~V}$ charged voltage using 3 Stage 2 Switch. Output was increased by $40 \mathrm{~W}$ when pulse repetition rate increased by $20 \mathrm{~Hz}$. Maximum $622 \mathrm{~W}$ was presented during $100 \mathrm{~Hz}$. Minimum output increase of $20 \%$ as observed when compared with the previous device.

\section{Conclusion}

As the new power device of transcranial magnetic stimulation, this study applies AVR one chip microprocessor and charge/discharge method as 3 Stage 2 Switch discharge method to research output control characteristics of transcranial magnetic stimulation according to changes in pulse repetition rate to draw the following conclusion. Due to the introduction of the 3 Stage 2 Switch discharge method, inexpensive commercial frequency condenser was used instead of the expensive high-frequency condenser to actualize under pricing of power device. One chip microprocessor was used to achieve real-time control of control motions through monitoring screen to setup motion waveforms from pulse 0 to pulse 6 on screen. Sequential charge/discharge was executed on 7 IGBT's after verifying signal waveform applied in gate to achieve precise control of transcranial magnetic stimulation. Stability and credibility of transcranial magnetic stimulation were verified from 20 to $100 \mathrm{~Hz}$ of the pulse repetition rate, and transcranial magnetic stimulation output was increased by $30 \mathrm{~W}$ to present a maximum of $628 \mathrm{~W}$ in $100 \mathrm{~Hz}$ during increase by $20 \mathrm{~Hz}$. Minimum output increase by $20 \%$ can be observed when compared with the previous device.

\section{Acknowledgement}

This work was supported by the Dong-A University Research Fund.

\section{References}

[1] V. Walsh and A. Pascual-Leone, Transcranial Magnetic Stimulation: a Neurochronometrics of Mind, The MIT Press, Cambridge (2005).

[2] E. Wassermann, Oxford Handbook of Transcranial Magnetic Stimulation, Oxford University Press, Oxford (2008).

[3] R. S. J. Frackowiak, K. J. Friston, C. Frith, et al. Human Brain Function, 2nd ed., Academic Press, San Diego (2003).

[4] H. B. Gak, Choi, S.-S., and Kim, W.-Y. J. Magnetics 16, 51 (2011).

[5] Orrin Devinsky, Aleksandar Beric. Eectrical and Magnetic Stimulation of the Brain and Spinal Cord, Raven Press, New York (2007).

[6] S. G. Mark, Transcranial Magnetic Stimulation in Clinical Psychiatry, American Psychiatric Publishing, Arlington (2006).

[7] A. L. Nicole, The Statiscal Analysis of Functional MRI Data, Springer Berlin (2008).

[8] S.-S. Choi and W.-Y. Kim, Journal of Biomedicine and Biotechnology 2011, 278062 (2011).

[9] Carl Senior. Method in Mind, The MIT Press, Cambridge (2009).

[10] Karl J. Friston. Statiscal Parametric Mapping, Academic Press, New York (2006).

[11] S.-S. Choi, S.-M. Lee, J.-H. Kim, and W.-Y. Kim, J. Magnetics 15, 213 (2010).

[12] W. Paulus, F. Tergau, M. A. Nitsche, and J. C. Rothwell Transcranial Magnetic Stimulation: Supplement to Clinical Neurophysiology Series, Volume 56, Elsevier; Amsterdam (2003). 